\title{
Avalanche phase conjugation
}

\author{
H. Ni and S. C. Rand \\ Division of Applied Physics, 1049 Randall Laboratory, University of Michigan, Ann Arbor, Michigan 48109-1120
}

Received May 15, 1992

\begin{abstract}
Image retrieval based on degenerate four-wave mixing with a new nonlinear mechanism is demonstrated. An avalanche nonlinearity associated with induced excited-state absorption is exploited to furnish phase conjugation with threshold behavior and potential motion sensitivity. Population pulsations are reported at high intensities.
\end{abstract}

Dynamic correction of distorted imagery by phase conjugation is one of many important applications of third-order processes in conventional nonlinear optics. Originally reported in the context of holography, ${ }^{1}$ phase conjugation has found widespread use in optical memories, ${ }^{2}$ laser power scaling, ${ }^{3}$ signal processing, ${ }^{4}$ spectroscopy, ${ }^{5}$ and other applications. ${ }^{6}$ The nonlinear mechanisms in earlier research involved resonant or nonresonant parts of the thirdorder susceptibility $\chi^{(3)}$ of independent atoms or of the susceptibility of atoms phased by fields such as the vibrational wave excitations in stimulated Raman and Brillouin scattering. Here, however, we report phase conjugation by a new mechanism based on delocalized, nonlinear dynamics of atoms in concentrated rare-earth crystals, with neighboring impurities interacting through their Coulomb fields.

Avalanche absorption on excited-state transitions in concentrated rare-earth crystals was first reported by Chivian et al. ${ }^{7}$ in $\mathrm{Pr}: \mathrm{LaCl}_{3}$ and studied by Case et $\mathrm{ll} .^{8}$ and others. ${ }^{9} \quad$ These authors applied rateequation concepts and analysis to model the induced excited-state absorption observed to increase as a function of incident intensity above a distinctive threshold intensity. We ${ }^{10}$ first pointed out the fundamental inconsistency of rate-equation descriptions in the presence of strong correlated dynamics of near-neighbor ions and furnished a simple densitymatrix theory to account not only for these correlations but for optical and interatomic coherences as well. We reported avalanche behavior at room temperature in Tm:YALO 3 and showed that the effect originates from internal dynamics consisting of two parts: correlated cross relaxation of Tm pairs followed by fast spatial energy migration, measured directly in recent experiments in our laboratory. The density-matrix equations account for the fact that nonlinear-optical response may originate from either the near-neighbor ion-ion coupling ${ }^{11}$ or the energy migration process, with the latter depending in a bilinear fashion on excited-state occupation probabilities of ions at the origin and the destination. In this Letter we present results of an experimental and theoretical investigation of the large third-order nonlinearity generated by avalanche dynamics in $\mathrm{Tm}: \mathrm{LiYF}_{4}$ crystals at room temperature.
The density-matrix description of avalanche dynamics provides the expression for the third-order polarization $P^{(3)}=\operatorname{Tr}\left[\mu \rho^{(3)}\right]$, which determines the degenerate four-wave mixing response, and hence the phase-conjugate signal intensity, in a straightforward way. ${ }^{12}$ In the pair basis of states used in Ref. 10, the steady-state solution is

$$
P^{(3)}=\frac{\mu_{52} V_{25}\left[\rho_{55}^{(3)}-\rho_{22}^{(3)}\right]}{i \hbar\left[\Gamma_{25}+i\left(\omega-\omega_{0}\right)\right]}+\text { c.c. }
$$

Here $\mu_{52}$ is the transition moment of the excitedstate optical transition between pair states 2 and 5 at frequency $\omega_{0}, V_{25}(t)=-\mu_{25} E(t)$ is the perturbation Hamiltonian that is due to optical field $E, \rho_{i j}$ is the population of the $j$ th pair state, and $\Gamma_{i j}$ is the dephasing rate between states $i$ and $j$. This equation shows the resonant nature of four-wave mixing on avalanche transitions and its proportionality to the population difference between the upper and lower states of the optical transition.

Avalanche absorption occurs in $\mathrm{Tm}: \mathrm{LiYF}_{4}$ at room temperature at relatively modest powers, as indicated in Fig. 1. Experiments were performed with a continuous-wave dye laser propagating perpendicular to the optic axis and tuned to the $\pi$-polarized ${ }^{3} F_{4}(2)-{ }^{1} G_{4}(2)$ (excited-state to excited-state) transition $^{13}$ at $648.4 \mathrm{~nm}$, a wavelength at which absorption normally does not occur. Transmission can be observed to decrease markedly above the onset of the avalanche process, the nature of which is shown schematically in the inset. Phase conjugation by degenerate four-wave mixing was achieved in a conventional geometry with counterpropagating pump beams and a probe wave incident at an external angle of $22^{\circ}$. A U.S. Air Force resolution target was placed in the probe beam as a test image. The probe beam subsequently passed through a beam splitter and aberrator and was tightly focused into the standing-wave region formed by the pump beams. Intensity and frequency of the balanced pump beams were adjusted until bright blue avalanche upconversion appeared in the crystal. Because of the abruptness of the avalanche in this crystal, it was possible to work extremely' close to threshold, in a regime such that either pump beam by itself was insufficient to sustain the avalanche, but both together resulted 


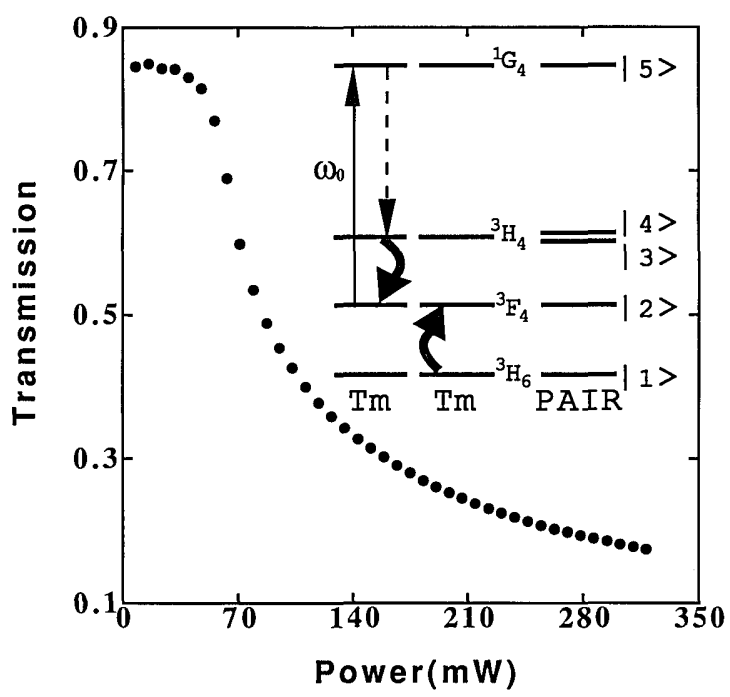

Fig. 1. Transmission of $7 \% \mathrm{Tm}^{3+}: \mathrm{LiYF}_{4}$ versus incident power at room temperature for an excitation wavelength of $\lambda_{\mathrm{ex}}=648.4 \mathrm{~nm}$. The focused beam radius was $47 \mu \mathrm{m}$. Inset: schematic of excited-state dynamics that give rise to induced absorption, together with the numbering scheme of the pair basis description from Ref. 10 .

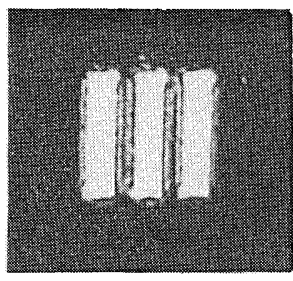

(a)

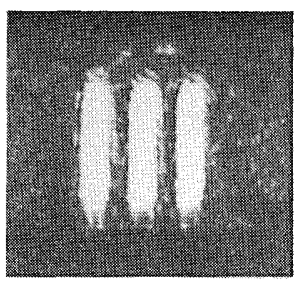

(c)

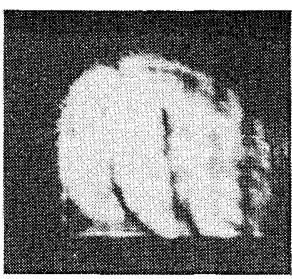

(b)

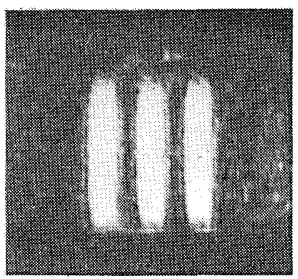

(d)
Fig. 2. Avalanche phase conjugation in $1.5 \% \mathrm{Tm}^{3+}: \mathrm{LiYF}_{4}$. (a) Unaberrated image of the resolution chart obtained with an ordinary mirror in place of the sample, (b) same image as in (a) with the aberrator in place, (c) restored image obtained with the conjugator and the aberrator in place, (d) phase-conjugate image from the sample with no aberrator. The spacing of the bars is 2 lines $/ \mathrm{mm}$.

in bright upconversion emission. Probe intensity was maintained at $20 \%$ of that of the individual pump-wave intensities for linearity.

Phase conjugation was observed in the backward probe direction as the complete restoration of a severely distorted bar image (Fig. 2). The best fidelity was obtained with a $1.5 \% \mathrm{Tm}: \mathrm{LiYF}_{4}$ sample, although $5 \%$ and $7 \%$ concentrations of Tm also produced phase conjugation. Crystals with less than approximately $0.5 \% \mathrm{Tm}$ are not expected to exhibit strong avalanche absorption. The higherconcentration crystals produced slightly distorted phase-conjugate images for reasons that are not completely understood but that appear to be related to thermal or self-focusing effects.

The dependence of phase-conjugate signal intensity on incident power is given in Fig. 3. Signal levels at low intensities were small until the full onset of avalanche dynamics at $160 \mathrm{~W} / \mathrm{cm}^{2}$ in each pump beam. The response below threshold arises from residual absorption in the far wings of ground-state resonances of Tm. Above the avalanche threshold point, the observed slope and signal level increase dramatically. When the steady-state solutions for populations in levels 2 and $5\left(\rho_{22}\right.$ and $\left.\rho_{55}\right)$ are calculated by including the small overlap with Tm ground-state absorptions (with an effective Einstein $B$ coefficient of $\left.B_{13}=8.7 \times 10^{-5} \mathrm{~cm}^{2} \mathrm{~W}^{-1} \mathrm{~s}^{-1}\right)$ and substituted into Eq. (1), the result is the solid curve in Fig. 3. Theory for the four-wave mixing interaction is seen to be in excellent agreement with experiment.

Finally, when the backward pump beam was chopped, its transmitted intensity and the phaseconjugate signal exhibited the behavior shown in Fig. 4. Overshoot occurred at the leading edge of the excitation pulse as predicted by numerical integration of the equations of Ref. 8. In addition, at an intensity of $6 \mathrm{~kW} / \mathrm{cm}^{2}$, small oscillations appeared in transmitted intensity. These additional features were reproduced by density-matrix theory incorporating spatial coherence, but power-dependent oscillations are not predicted by rate-equation analysis that omits all coherences. The observed oscillations may therefore be related to heavily damped, coherent delocalization of excitation between two Tm ions.

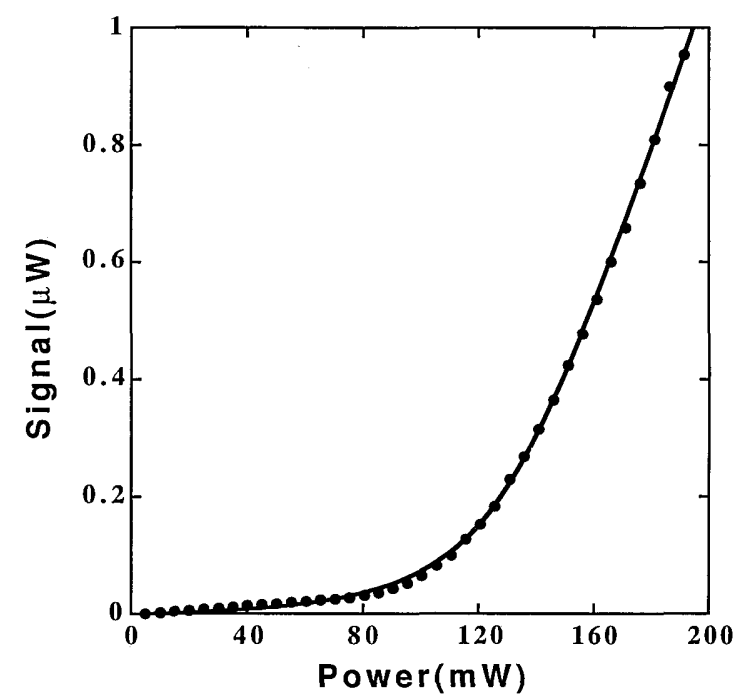

Fig. 3. Avalanche phase-conjugate signal versus total input power in $1.5 \% \mathrm{Tm}^{3+}: \mathrm{LiYF}_{4}$ for a focal spot of radius $96 \mu \mathrm{m}$. The solid curve is a least-squares fit based on Eq. (1). Fixed theoretical parameters ${ }^{10}$ determined by previous experiments ${ }^{14}$ were $\gamma_{2}=\gamma_{3}=62.5 \mathrm{~s}^{-1}, \gamma_{4}=$ $690 \mathrm{~s}^{-1}, \gamma_{5}=8333 \mathrm{~s}^{-1}$, and $\lambda=1.4 \times 10^{-10} \mathrm{~s}^{-1}$. In the absence of direct measurements of dephasing or radiative lifetime on the $5 \rightarrow 2$ transition, the product of $\tau$ and $\Gamma_{25}$ was treated as a scaling parameter (best-fit value of $\left.4.43 \times 10^{6}\right)$. Best-fit values for the migration rate and intrapair relaxation term were $\alpha=2 \times 10^{5} \mathrm{~s}^{-1}$ and $\left|H_{34}\right|^{2} / \hbar^{2} \Gamma_{34}=810 \mathrm{~s}^{-1}$. 

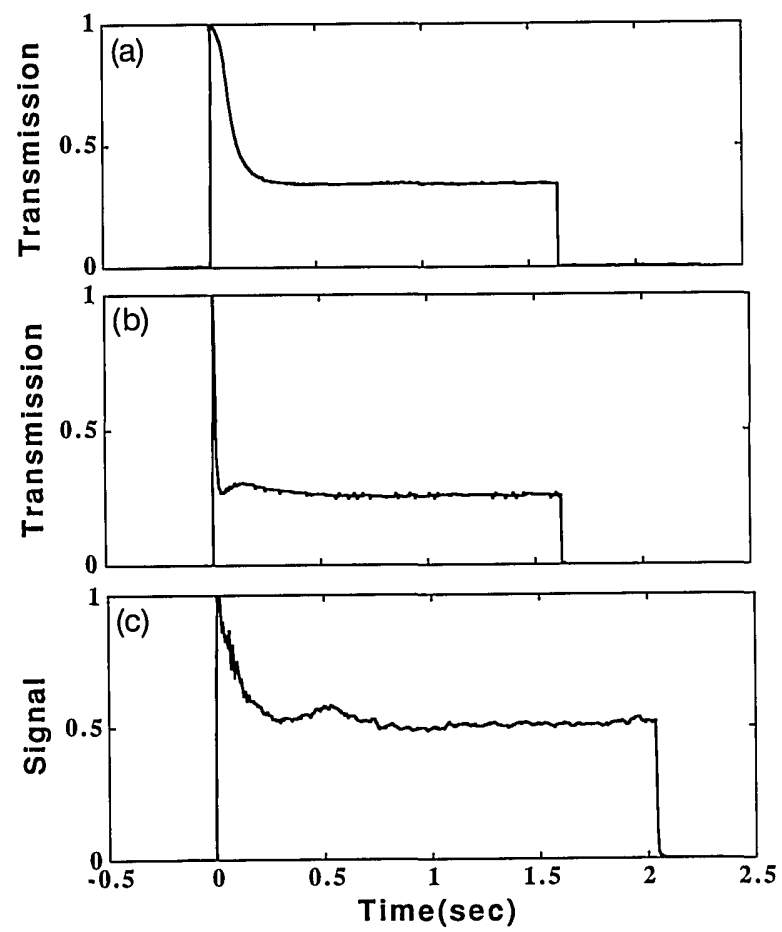

Fig. 4. (a) Transmission versus time for square pulse excitation of $5 \% \mathrm{Tm}^{3+}: \mathrm{LiYF}_{4}$ with an incident intensity of $7.6 \times 10^{2} \mathrm{~W} / \mathrm{cm}^{2}$. (b) Transmission versus time at high intensity $\left(6.1 \times 10^{3} \mathrm{~W} / \mathrm{cm}^{2}\right)$; notice the decreased rise time of induced absorption and the appearance of population oscillations. (c) Phase-conjugate signal intensity versus time for an effective pump intensity $\left(I_{1} I_{2}\right)^{1 / 2}=1.3 \times$ $10^{3} \mathrm{~W} / \mathrm{cm}^{2}$; again, population oscillations are evident.

In the context of image processing, the overshoot feature is quite interesting. It shows that enhancement of the phase-conjugate signal arises from points of the image field in which the optical intensity is initially low but crosses the avalanche threshold as intensity increases with time. With a strong probe wave or with the backward pump made into the image-bearing beam, this effect should appear in the signal wave in a manner useful for detection of motion in an otherwise stationary target image. Intensity steps at the front edges of moving objects should give particularly bright image lines, which produces an enhanced outline. This temporal edge enhancement, or avalanche halo, should appear for intensity variations faster than the avalanche response time, which is roughly $50 \mathrm{~ms}$ near threshold in the case of $\mathrm{Tm}: \mathrm{LiYF}_{4}$. In combination with the adjustable thresholding capability of avalanche phase conjugation, this novelty filtering ${ }^{15}$ aspect of avalanche dynamics should permit unique imageprocessing capabilities.

In summary, we have demonstrated image phase conjugation at visible wavelengths using an avalanche nonlinearity. In addition to permitting conjugation at new (excited-state) transition wavelengths in rare-earth solids, the avalanche mechanism introduces a threshold not present in other straightforward forms of phase conjugation. Another interesting feature of avalanche phase con- jugation is the enhancement of signals or reconstructed images from probe waves undergoing intensity variations that should generate temporal edge enhancement in reconstructed dynamic images. The experimental magnitude and threshold of the steady-state third-order nonlinearity in $\mathrm{Tm}^{3+}: \mathrm{LiYF}_{4}$ can be accounted for by the energy migration mechanism (incoherent delocalization of excitation). Further research is proceeding on oscillations in the transient response that may be related to near-neighbor dynamics (coherent delocalization).

The authors gratefully acknowledge sponsorship by the U.S. Air Force Office of Scientific Research. $\mathrm{H}$. Ni thanks the Barbour Scholarship Program for graduate support. We are also indebted to N. Cockcroft and R. M. MacFarlane for the loan of several samples.

\section{References}

1. E. N. Leith and J. Upatnieks, J. Opt. Soc. Am. 52, 1123 (1962); SPIE J. 4, 3 (1965); H. Kogelnik, Bell Syst. Tech. J. 44, 2451 (1965).

2. G. J. Dunning, E. Marom, Y. Owechko, and B. H. Soffer, Opt. Lett. 12, 346 (1987); S. Kröll, L. Jusinski, and R. Kachru, Opt. Lett. 16, 517 (1991).

3. O. Y. Nosach, V. I. Popovichev, V. V. Ragul'skii, and F. S. Faizullov, JETP Lett. 16, 435 (1972); D. Rockwell, IEEE J. Quantum Electron. 24, 1124 (1988).

4. S. Weiss, S. Sternklar, and B. Fischer, Opt. Lett. 12, 114 (1987).

5. D. Redman, S. W. Brown, R. H. Sands, and S. C. Rand, Phys. Rev. Lett. 67, 3420 (1991).

6. R. A. Fisher, ed., Optical Phase Conjugation (Academic, New York, 1983).

7. J. Chivian, W. Case, and D. Eden, Appl. Phys. Lett. 35, 124 (1979).

8. W. E. Case, M. E. Koch, and A. W. Kueny, J. Lumin. 45, 351 (1990).

9. N. J. Krasutsky, J. Appl. Phys. 54, 1261 (1983); A. W. Kueny, W. E. Case, and M. E. Koch, J. Opt. Soc. Am. B 6, 639 (1989); N. Pelletier-Allard and R. Pelletier, J. Lumin. 46, 217 (1990); W. Lenth and R. M. Macfarlane, J. Lumin. 45, 346 (1990); N. PelletierAllard and R. Pelletier, Opt. Commun. 81, 247 (1991); U. Oetliker, M. J. Riley, P. S. May, and H. U. Gudel, Coord. Chem. Rev. 111, 125 (1991).

10. H. Ni and S. C. Rand, Opt. Lett. 16, 1424 (1991).

11. J. Rai and C. M. N. Bowden, in Digest of International Quantum Electronics Conference (Optical Society of America, Washington, D.C., 1990), paper QTuN3.

12. See, for example, S. C. Rand, in Lasers, Spectroscopy and New Ideas, W. M. Yen and M. D. Levenson, eds., Vol. 54 of Springer Series in Optical Sciences (Springer-Verlag, Berlin, 1987), pp. 268-289.

13. H. P. Jenssen, A. Linz, R. P. Leavitt, C. A. Morrison, and D. E. Wortman, Phys. Rev. B 11, 92 (1975).

14. A. Brenier, J. Rubin, R. Moncorge, and C. Pedrini, J. Phys. (Paris) 50, 1463 (1989).

15. D. Z. Anderson, D. M. Lininger, and J. Feinberg, Opt. Lett. 12, 123 (1987); a recent bibliography on novelty filtering may be found in C. Soutar, C. M. Cartwright, W. A. Gillespie, and Z. Q. Wang, Opt. Commun. 86, 255 (1991). 